\title{
Is poststroke depression the same as major depression?
}

\author{
Paul R. Albert, PhD
}

\section{What causes PSD versus major depression?}

In the United States, it is estimated that 11 million strokes occurred within 1 year (1998). ${ }^{1,2}$ Poststroke depression (PSD) is a common outcome following stroke, with $30 \%$ of patients poststroke having comorbid major depression and $80 \%$ of patients having milder forms of depression. ${ }^{3,4}$ Patients with PSD show increased likelihood of recurrent stroke and depression, and increased mortality compared with nondepressed patients. ${ }^{5}$ Major depression is a chronic disorder that is often comorbid with anxiety. ${ }^{6}$ Environmental stressors, including having a stroke, contribute to predisposition and can trigger episodes of major depression. ${ }^{6}$ Both PSD and major depression are diagnosed according to the same set of criteria that combine diverse and sometimes opposite symptoms, which contributes to the heterogeneous nature of major depression. ${ }^{7}$ One of the goals of psychiatry today is to move toward precision medicine by elucidating biomarkers for better characterization of mental illness and its treatment. ${ }^{8}$ Identifying the underlying molecular, cellular or circuit changes leading to behavioural phenotypes can be used to target therapeutic interventions to specific mechanisms driving mental illness. This raises the question of whether PSD and major depression should be considered the same or separate mental illnesses.

Poststroke depression appears to be triggered by or linked to the ischemic event, as depression occurs within three months of a stroke. ${ }^{3}$ How stroke triggers depression is unclear. The sudden physical handicap and loss of personal independence following stroke may trigger a chronic state of depression. However, there is evidence that lesions associated with stroke or vascular ischemic events, particularly in cases of covert stroke where no evident impairment presents, can lead to depression. The size, number of ischemic lesions, and whether the lesions disrupt the midbrain, limbic and medial prefrontal cortical (mPFC) circuitry are implicated in depression. ${ }^{9}$ In particular, white matter lesions are associated with metabolic alterations in this circuitry ${ }^{10}$ and are correlated with major depression. ${ }^{11}$ Several independent studies have indicated that in patients with ischemic stroke, lesions in the left frontal cortex, left basal ganglia or brainstem are correlated with affective and apathetic symptoms of depression. ${ }^{2,13}$
Thus, an emerging concept that remains to be tested is that strokes leading to PSD may disrupt the anxiety or depression circuitry at several locations. ${ }^{14}$

\section{Detecting PSD and vascular depression}

Poststroke depression is a form of vascular depression related to large vessel occlusion. ${ }^{14}$ Covert (or "silent") strokes include small brain infarcts or microbleeds (lacunae or white matter infarctions) that do not result in obvious sensorimotor impairments. Covert strokes are five-fold more frequent forms of stroke, with one stroke every nine minutes (62 000/yr) and one covert stroke each two minutes in Canada. ${ }^{2,15}$ Recently, vascular depression has been associated with the presence of covert strokes. ${ }^{11}$ Thus, the prevalence of vascular depression is potentially five-fold higher than that of PSD. ${ }^{16}$ On the other hand the incidence of covert stroke in depressed patients is $46 \%,{ }^{2}$ consistent with a strong bidirectional connection between the two. ${ }^{17}$ However, the "silence" of covert stroke presents a challenge in detecting vascular versus major depression. Presently, covert stroke is often detected only once a more severe condition arises requiring imaging, at which time the presence of lacunae or white matter hyperintensities may be evident. Typically, patients presenting with depression are not referred for imaging, but the need for imaging may be important, particularly in patients with risk factors for cerebrovascular disease (e.g., cardiovascular disease, hypertension, diabetes). ${ }^{2,17}$ Recently, imaging has been applied with repetitive transcranial magnetic stimulation (rTMS), which is recommended as second-line treatment for major depression in patients who do not respond to antidepressant treatment. ${ }^{18}$ It remains unclear what proportion of patients with major depression show stroke pathology that may suggest vascular depression. Based on the prevalence of covert stroke, this proportion may be substantial.

\section{Does PSD/vascular depression respond to antidepressant treatment?}

Assuming we can distinguish PSD and vascular depression from major depression, how does this impact treatment? It remains unclear. Like major depression, PSD is mainly

Correspondence to: P. Albert, UOttawa Brain and Mind Research Institute, Ottawa Hospital Research Institute, 451 Smyth Road, Ottawa, ON K1H 8M5; paul.Albert@uottawa.ca

DOI: 10.1503/jpn.180015 
treated with antidepressants, such as selective serotonin reuptake inhibitors (SSRIs). ${ }^{14}$ There is evidence that in addition to improving anxiety and depression symptoms, SSRIs also enhance poststroke cognitive ability and recovery..$^{9,14,19}$ However, most antidepressants require chronic treatment (3-4 weeks) to produce improvement. ${ }^{20}$ For major depression, the STAR*D study found that although about $50 \%$ of patients responded to SSRIs, remission occurred in only $30 \%$. ${ }^{21}$ It remains unclear whether the same can be said for PSD or vascular depression; notably, chronic SSRI treatment increases the risk of hemorrhage. ${ }^{22,23}$ However, to examine treatment outcomes for vascular depression, it will be necessary to first detect it using imaging, as mentioned earlier.

What can preclinical studies tell us about treatment for PSD? Most rodent models of PSD have used middle cerebral artery occlusion, which produces a large and variable lesion, leading to sensorimotor impairments and altered behaviour. ${ }^{24}$ The sensorimotor impairments may confound behavioural tests that are often based on intact sensorimotor function. Thus, in order to obtain a robust depression phenotype, middle cerebral artery occlusion has been combined with chronic stress, which itself induces depression-like behaviour. Although chronic SSRI treatment is effective, this raises the question of whether stroke- or stress-induced depression is being studied. One recent study used photothrombosis to elicit an anterior cortical stroke and waited until sensorimotor function recovered before testing behaviour. ${ }^{25}$ The researchers showed that chronic SSRI treatment is effective to reverse the anxiety- and depression-like phenotypes, involving increase in hippocampal brain-derived neurotrophic factor. ${ }^{25}$ Another model used endothelin-1 microinjection to induce a small lesion of the left medial prefrontal cortex, resulting in anxietyand depression-like behaviour, as well as cognitive impairment, with no sensorimotor impairment. ${ }^{26,27}$ In this model, chronic SSRI treatment, but not free running wheel exercise, reversed the behavioural and cognitive phenotypes. Interestingly, the small lesion became refilled with neurons, which may be recruited by SSRI treatment. These findings suggest that chronic SSRI treatment is effective to reverse PSD, in part through growth factor induction. In this regard, chronic SSRI treatment may trigger neuroplasticity to promote recovery from depression, including PSD. However, clinical studies of the effectiveness of SSRI treatment in human PSD or vascular depression need to be done and will require a dedicated pretreatment imaging screen to detect vascular lesions in drug-naive, depressed patients.

\section{Etiology of PSD and depression: inflammation link?}

The pathology underlying PSD and major depression differs. Stroke leads to neuronal and glial cell death due to hypoxic lesion, whereas major depression is not always associated with detectable cell death. However, postmortem studies of brains from depressed individuals who committed suicide show loss of glia and neurons. ${ }^{28,29}$ Chronic stress is known to induce neuronal atrophy, ${ }^{30}$ particularly in the hippocampus, and reductions in hippocampal volume can be seen in depressed humans. ${ }^{31}$ It has been suggested that neuroinflammatory processes involving cytokine and microglial mobilization underlie some forms of depression, ${ }^{29,32}$ and inflammatory processes may be important in the pathology of PSD and vascular depression. ${ }^{33,34}$ Therefore, vascular depression may fall into a "neuroinflammatory" depression phenotype. ${ }^{14}$

Hence the question remains: how much of major depression is really undiagnosed PSD? It can be argued that increased effort to diagnose both systemic inflammation and small-vessel disease in depressed individuals is warranted, since many of the risk factors leading to stroke (e.g., diabetes, atherosclerosis, stroke, heart failure) are also tied to depression. ${ }^{2,16,35}$ The good news is that these risk factors, if recognized early enough, can be controlled to prevent or reverse the depression phenotype. ${ }^{36}$

Acknowledgements: The author thanks Drs. Faranak Vahid-Ansari, Antoine M. Hakim, and the members of the JPN Editorial Board for their insightful comments and suggestions. P. Albert is supported by grants from the Canadian Institutes of Health Research and the HSF Canadian Partnership for Stroke Recovery.

Affiliation: From the UOttawa Brain and Mind Research Insitute, Ottawa Hospital Research Institute, University of Ottawa, Ottawa, Ont., Canada.

Competing interests: None declared.

\section{References}

1. Leary MC, Saver JL. Annual incidence of first silent stroke in the United States: a preliminary estimate. Cerebrovasc Dis 2003;16:280-5.

2. Vermeer SE, Longstreth WT Jr, Koudstaal PJ. Silent brain infarcts: a systematic review. Lancet Neurol 2007;6:611-9.

3. Paolucci S, Gandolfo C, Provinciali L, et al. The Italian multicenter observational study on post-stroke depression (DESTRO). J Neurol 2006;253:556-62.

4. Hackett ML, Pickles K. Part I: frequency of depression after stroke: an updated systematic review and meta-analysis of observational studies. Int J Stroke 2014;9:1017-25.

5. Ayerbe L, Ayis S, Crichton S, et al. The natural history of depression up to 15 years after stroke: the South London Stroke Register. Stroke 2013;44:1105-10.

6. Hyman S. Mental illness: genetically complex disorders of neural circuitry and neural communication. Neuron 2000;28:321-3.

7. Krishnan V, Nestler EJ. The molecular neurobiology of depression. Nature 2008;455:894-902.

8. Insel TR, Cuthbert BN. Medicine. Brain disorders? Precisely. Science 2015;348:499-500.

9. Flaster M, Sharma A, Rao M, et al. Poststroke depression: a review emphasizing the role of prophylactic treatment and synergy with treatment for motor recovery. Top Stroke Rehabil 2013;20:139-50.

10. Capizzano AA, Jorge RE, Robinson RG. Limbic metabolic abnormalities in remote traumatic brain injury and correlation with psychiatric morbidity and social functioning. J Neuropsychiatry Clin Neurosci 2010;22:370-7.

11. Taylor WD, Aizenstein HJ, Alexopoulos GS. The vascular depression hypothesis: mechanisms linking vascular disease with depression. Mol Psychiatry 2013;18:963-74.

12. Terroni L, Amaro E, Iosifescu DV, et al. Stroke lesion in cortical neural circuits and post-stroke incidence of major depressive episode: a 4-month prospective study. World J Biol Psychiatry 2011; 12:539-48.

13. Murakami T1, Hama S, Yamashita H, et al. Neuroanatomic pathways associated with poststroke affective and apathetic depression. Am J Geriatr Psychiatry 2013;21:840-7.

14. Robinson RG, Jorge RE. Post-stroke depression: a review. Am J Psychiatry 2016;173:221-31.

15. Black S, Butcher K, Chambers LW, et al. Mind the connection. Preventing stroke and dementia. 2016 stroke report. Ottawa (ON): Heart and Stroke 
Foundation of Canada; 2016. Available: www.strokebestpractices.ca/ wp-content/uploads/2016/06/HSF_StrokeReport2016_EN-Rev-1.pdf (accessed 2018 Jan. 31).

16. Brookes RL, Herbert V, Lawrence AJ, et al. Depression in smallvessel disease relates to white matter ultrastructural damage, not disability. Neurology 2014;83:1417-23.

17. Hakim AM. Depression, strokes and dementia: new biological insights into an unfortunate pathway. Cardiovasc psychiatry neurol 2011;2011:649629.

18. Milev RV, Giacobbe P, Kennedy SH, et al. Canadian Network for Mood and Anxiety Treatments (CANMAT) 2016 clinical guidelines for the management of adults with major depressive disorder: section 4. Neurostimulation treatments. Can J Psychiatry 2016; 61:561-75.

19. Chollet F, Tardy J, Albucher JF, et al. Fluoxetine for motor recovery after acute ischaemic stroke (FLAME): a randomised placebocontrolled trial. Lancet Neurol 2011;10:123-30.

20. Mead GE, Hsieh CF, Lee R, et al. Selective serotonin reuptake inhibitors (SSRIs) for stroke recovery. Cochrane Database Syst Rev 2012;11:Cd009286

21. Rush AJ, Warden D, Wisniewski SR, et al. STAR ${ }^{*} \mathrm{D}$ : revising conventional wisdom. CNS Drugs 2009;23:627-47.

22. Mortensen JK, Larsson H, Johnsen SP, et al. Impact of prestroke selective serotonin reuptake inhibitor treatment on stroke severity and mortality. Stroke 2014;45:2121-3.

23. Scheitz JF, Turc G, Kujala L, et al. Intracerebral hemorrhage and outcome after thrombolysis in stroke patients using selective serotonin-reuptake inhibitors. Stroke 2017;48:3239-44.

24. Kronenberg G, Gertz K, Heinz A, et al. Of mice and men: modelling post-stroke depression experimentally. Br J Pharmacol 2014; 171:4673-89.
25. Jin HI, Pei L, Li YN, et al. Alleviative effects of fluoxetine on depressivelike behaviors by epigenetic regulation of BDNF gene transcription in mouse model of post-stroke depression. Sci Rep 2017;7:14926.

26. Vahid-Ansari F, Albert PR. Chronic fluoxetine induces activity changes in recovery from poststroke anxiety, depression, and cognitive impairment. Neurotherapeutics 2017.

27. Vahid-Ansari F, Albert PR et al. Persistent post-stroke depression in mice following unilateral medial prefrontal cortical stroke. Transl Psychiatry 2016;6:e863.

28. Rajkowska G, Miguel-Hidalgo JJ, Wei J, et al. Morphometric evidence for neuronal and glial prefrontal cell pathology in major depression. Biol Psychiatry 1999;45:1085-98.

29. Mechawar N, Savitz J. Neuropathology of mood disorders: Do we see the stigmata of inflammation? Transl Psychiatry 2016;6:e946.

30. McEwen BS. Stress and hippocampal plasticity. Annu Rev Neurosci 1999;22:105-22.

31. MacQueen GM, Campbell S, McEwen BS, et al. Course of illness, hippocampal function, and hippocampal volume in major depression. Proc Natl Acad Sci U S A 2003;100:1387-92.

32. Anisman H. Cascading effects of stressors and inflammatory immune system activation: implications for major depressive disorder I Psychiatry Neurosci 2009;34:4-20.

33. Anrather J, Iadecola C. et al. Inflammation and stroke: an overview. Neurotherapeutics 2016;13:661-70.

34. Dinan TG. Inflammatory markers in depression. Curr Opin Psychiatry 2009;22:32-6

35. Evans DL, Charney DS, Lewis L et al. Mood disorders in the medically ill: scientific review and recommendations. Biol Psychiatry 2005; 58:175-89.

36. Hakim AM. Perspective: silent, but preventable, perils. Nature 2014;510:S12.

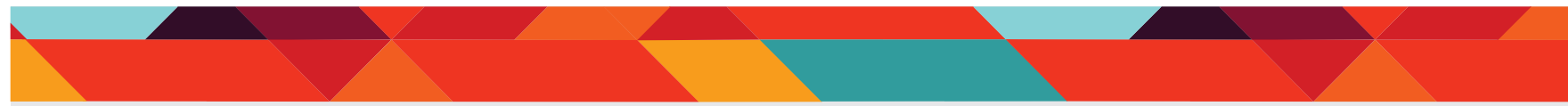

\section{Contact Sheridan Press 1-800-635-7181 ext. 8065 marcus.glover@sheridan.com www.sheridan.com/cma/eoc}
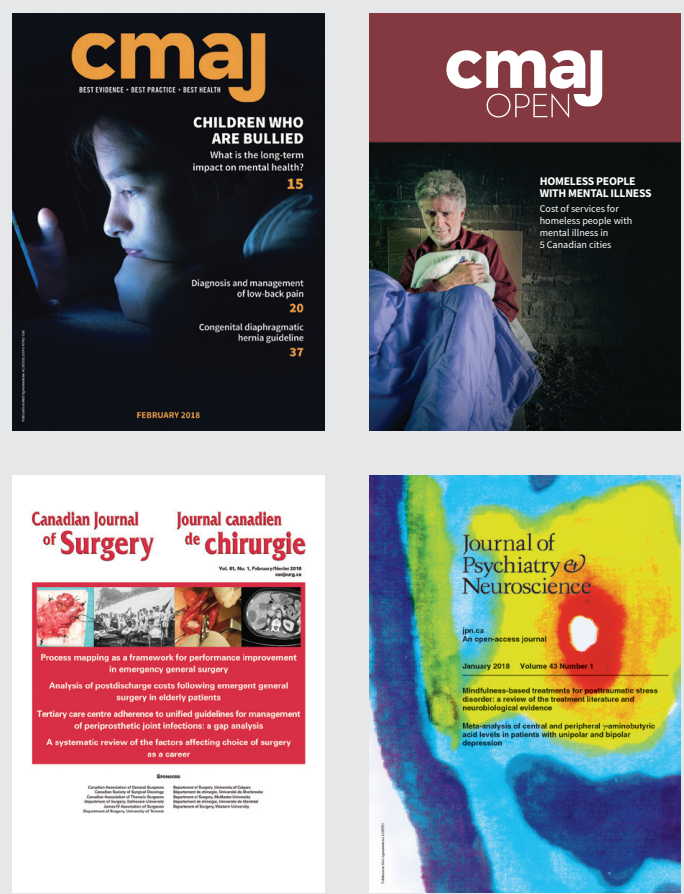